\title{
Morse index of Steady State Bifurcation for a Class of Reaction-diffusion Equations
}

\author{
Yupei Zhang ${ }^{1}$, Xibing $\mathrm{He}^{2}$ \\ ${ }^{1}$ Nanchang Institute of Science \& Technology, Nanchang, 330108, China \\ ${ }^{2}$ Department of Mathematics and Computer Science, Nanchang Normal University, Nanchang, 330032, \\ China
}

Keywords: Morse index, double eigenvalue, Reaction-diffusion equations, steady-state bifurcation

\begin{abstract}
In this paper, we compute the Morse index of the steady-state bifurcation solutions from the eigenvalue $\lambda=\lambda_{9}$ with multiplicity two of the Laplacian operator for a class of reaction-diffusion equations with Robin boundary conditions on the two-dimensional rectangular area $[0,2 \pi] \times[0, \pi]$.
\end{abstract}

\section{Introduction}

In bifurcation theory, people are not only concerned with the branch structure of the solutions when the parameters of nonlinear bifurcation problem change, but also concerned about the stability of the solutions. The introduction of the Morse index is used to determine the stability of the solutions. Therefore, a discussion for the Morse index has a very important role in the stability theory. It is generally difficult to determine the Morse exponent of the bifurcation solution directly from the operator. From the stability theory of the dynamic system, it is known that when the Morse exponent of a non-degenerate bifurcation solution is 0 , it is a local asymptotically stable equilibrium point. But if its Morse exponent is greater than 1, then it's a saddle point.

Study ${ }^{[3]}$ local bifurcation from the branch of trivial solutions for a class of semilinear elliptic equations, at the second eigenvalue $\lambda_{2}$ of a square, and also compute the Morse index of the bifurcation solutions obtained earlier. Based on the bifurcation theorem of Crandall and Rabinowitz, taking the variation of the local bifurcation of a single curve in nonlinear equation as the research content, and combining the global bifurcation theory ${ }^{[4]}$ get many conclusions about the exact solution number of semilinear elliptic equations.

\section{Preliminaries}

In the last paper ${ }^{[5]}$, we restrict ourselves in what follows to a special case of the reaction-diffusion equation

$$
\left\{\begin{array}{lr}
\frac{\partial u}{\partial t}=\Delta u+\lambda u+f(\bar{x}, u) & \text { in } \bar{x}=(x, y) \in \Omega \\
u(0, y, t)=u(2 \pi, y, t)=0 & \forall y \in[0, \pi], t>0 \\
\frac{\partial u}{\partial n}(x, 0, t)=\frac{\partial u}{\partial n}(x, \pi, t)=0 & \forall x \in[0,2 \pi], t>0 \\
u(\bar{x}, 0)=u_{0}(\bar{x}) & t=0
\end{array}\right.
$$

where $t \in[0,+\infty), \lambda \in R^{1}$ and $f$ satisfies the two following conditions:

$$
\text { (i) } f \in C^{3}\left(\bar{\Omega} \times R^{1}\right) \text {; (ii) } f(\bar{x}, 0)=f_{u}(\bar{x}, 0)=f_{\text {ии }}(\bar{x}, 0)=0, f_{\text {иии }}(\bar{x}, 0)=k \neq 0 \text {. }
$$

It is easy to see that the problem of steady state bifurcation from the double eigenvalue in above equation can be converted into the corresponding problem of the following semi-linear elliptic 
equation:

$$
\left\{\begin{array}{lr}
\Delta u+\lambda u+f(\bar{x}, u)=0 & \text { in } \bar{x}=(x, y) \in \Omega \\
u(0, y)=u(2 \pi, y)=0 & \forall y \in[0, \pi] \\
\frac{\partial u}{\partial n}(x, 0)=\frac{\partial u}{\partial n}(x, \pi)=0 & \forall x \in[0,2 \pi]
\end{array}\right.
$$

Through Taylor expansion, we can find that $f(\bar{x}, u)=u^{3}(1+\theta(\bar{x}, u))$ for $k>0$; accordingly we consider $f(\bar{x}, u)=-u^{3}(1+\theta(\bar{x}, u))$ for $k<0$.Also we have found the conclusions as follows:

Lemma 1 For the eigenvalue problem of a two-dimensional case:

$$
\left\{\begin{array}{lr}
\Delta u+\lambda u=0 & \text { in }(x, y) \in \Omega \\
u(0, y)=u(2 \pi, y)=0 & \forall y \in[0, \pi] \\
\frac{\partial u}{\partial n}(x, 0)=\frac{\partial u}{\partial n}(x, \pi)=0 & \forall x \in[0,2 \pi]
\end{array}\right.
$$

In the Fourier coordinate system, the eigenvalues and the eigenfunctions of the Laplacian operator are expressed as follows:

$$
\left\{\begin{array}{l}
\lambda_{m, n}=\frac{n^{2}}{4}+m^{2} \\
u_{m, n}(x, y)=\tilde{c} \sin \frac{n}{2} x \cos m y
\end{array}\right.
$$

where $m \in N, n \in N^{+}, \tilde{c}$ is a nonzero constant. In particular, the dual eigenvalue is $\lambda_{9}=\lambda_{2,2}=\lambda_{1,4}=5$.

On the basis of Lemma 1, we label $A=\sin x \cos 2 y$ and $B=\sin 2 x \cos y$ as all eigenfunctions associated to the double eigenvalue $\lambda_{9}=\lambda_{2,2}=\lambda_{1,4}=5$.After that we introduce the normalized eigenfunction $\phi_{\alpha}(x, y)=\cos \alpha A+\sin \alpha B, \alpha \in[0,2 \pi)$, which is a parametric representation of all eigenfunctions $\phi$ with $\|\phi\|_{L^{2}}=\pi / \sqrt{2}$. Alongside with $\phi_{\alpha}$ we introduce an orthogonal eigenfunction defined by $\psi_{\alpha}(x, y)=\sin \alpha A-\cos \alpha B$.

Theorem 1 Let $\Omega=[0,2 \pi] \times[0, \pi] \subset R^{2}$.Then there exist an $\varepsilon>0$ and a neighbourhood $U$ of $\left(\lambda_{9}, 0\right)$ in $R^{1} \times C(\bar{\Omega})$ such that the set of all bifurcation solutions of (1) in $U$ can be described as the union of four $C^{1}$ curves: $s \in(-\varepsilon, \varepsilon) \mapsto\left(\lambda_{i}(s), u_{i}(s)\right), i=1, \cdots, 4$, such that

$$
\left\{\begin{array}{l}
\lambda_{i}(s)=\lambda_{9}+\sigma_{i} s^{2}+o\left(s^{2}\right) \\
u_{i}(s)=s \phi_{\alpha_{i}}+o(s)
\end{array}\right.
$$

where $\alpha_{1}=0, \alpha_{2}=\pi / 4, \alpha_{3}=\pi / 2, \alpha_{4}=3 \pi / 4$, and

$$
\begin{gathered}
\sigma_{1}=\sigma_{3}=-9 / 16, \sigma_{2}=\sigma_{4}=-21 / 32, \text { for } k>0 \\
\sigma_{1}=\sigma_{3}=9 / 16, \sigma_{2}=\sigma_{4}=21 / 32, \text { for } k<0 .
\end{gathered}
$$

\section{The Main Results}

On the basis of the former work, in this section we compute the Morse index of the solutions 
corresponding to the four bifurcating branches found in Theorem 1.We specify that by Morse index of a solution of (1) we mean the number of negative eigenvalues of its linear problem (see, e. g.,[3,4]).Thus we need to find the number of negative eigenvalues $\mu$ of the linearized equation:

$$
\left\{\begin{array}{lr}
\Delta \omega+\lambda \omega \pm u^{2}\left(3(1+\theta(\bar{x}, u))+u \theta_{u}(\bar{x}, u)\right) \omega+\mu \omega=0 & \text { in } \Omega \\
\omega(0, y)=\omega(2 \pi, y)=0 & \forall y \in[0, \pi] \\
\frac{\partial \omega}{\partial n}(x, 0)=\frac{\partial \omega}{\partial n}(x, \pi)=0 & \forall x \in[0,2 \pi]
\end{array}\right.
$$

Theorem 2 If or , the Morse index of the bifurcation solution curves cross of (1) was 10 for , and 8 for ;If or , the Morse index of the stationary bifurcation solution curves of (1) was 9,both and .

Proof. According to the conclusion of Theorem 1, we can set, substituting them into the above equation and simplifying we obtain:

$$
\left\{\begin{array}{lr}
\Delta \omega+\lambda_{9} \omega+s^{2} \sigma \omega \pm 3 s^{2}\left(\phi_{\alpha}+s^{2} \psi\right)^{2}\left(1+\theta\left(\bar{x}, s \phi_{\alpha}+s^{3} \psi\right)\right) \omega & \\
\pm s^{3}\left(\phi_{\alpha}+s^{2} \psi\right)^{3} \theta_{u}\left(\bar{x}, s \phi_{\alpha}+s^{3} \psi\right) \omega+\mu \omega=0 & \text { in } \Omega \\
\omega(0, y)=\omega(2 \pi, y)=0 & \forall y \in[0, \pi] \\
\frac{\partial \omega}{\partial n}(x, 0)=\frac{\partial \omega}{\partial n}(x, \pi)=0 & \forall x \in[0,2 \pi]
\end{array}\right.
$$

where now $\mu=\mu(s), \omega=\omega(s)$.We normalize $\omega$ by $\|\omega\|_{L^{2}}=\frac{\pi}{\sqrt{2}}$. As $s$ goes to zero, it follows that $\mu(s)$ and $\omega(s)$ respectively converge to an eigenvalue and eigenfunction of the problem below

$$
\left\{\begin{array}{l}
\Delta \omega+\lambda_{9} \omega+\mu \omega=0 \\
\omega(0, y)=\omega(2 \pi, y) w=0 \\
\frac{\partial \omega}{\partial n}(x, 0)=\frac{\partial \omega}{\partial n}(x, \pi)=0
\end{array}\right.
$$

whose set of eigenvalues is

$$
\left\{\lambda_{1}-\lambda_{9}, \lambda_{2}-\lambda_{9}, \lambda_{3}-\lambda_{9}, \lambda_{4}-\lambda_{9}, \lambda_{5}-\lambda_{9}, \lambda_{6}-\lambda_{9}, \lambda_{7}-\lambda_{9}, \lambda_{8}-\lambda_{9}, 0, \lambda_{10}-\lambda_{9}, \cdots\right\} .
$$

Thus for $s$ small ,the first eight eigenvalues of (2) are negative and close to $\lambda_{1}-\lambda_{9}, \lambda_{2}-\lambda_{9}, \lambda_{3}-\lambda_{9}$, $\lambda_{4}-\lambda_{9}, \lambda_{5}-\lambda_{9}, \lambda_{6}-\lambda_{9}, \lambda_{7}-\lambda_{9}, \lambda_{8}-\lambda_{9}$. There is also one positive eigenvalue close to $\lambda_{10}-\lambda_{9}$, and we have to decide what happens with the eigenvalues close to 0 . Notice that for small, there are always two such eigenvalues $\mu_{1}(s), \mu_{2}(s)$ counting multiplicity, since the proper subspace has to be two dimensional. Let $\omega_{1}(s), \omega_{2}(s)$ denote the orthogonal eigenfunctions corresponding to these two eigenvalues. Then $\omega_{i}(s) \rightarrow A_{i} \phi_{\alpha}+B_{i} \psi_{\alpha}$ as $s \rightarrow 0$, where $i=1,2, A_{i}^{2}+B_{i}^{2}=1$ and $A_{1} A_{2}+B_{1} B_{2}=0$.

Multiplying the first formula of (2) by $\phi_{\alpha}$, taking $\phi_{\alpha}, \psi_{\alpha} \in M$ into consideration and integrating over over $\Omega$, the first two terms of (2) can be converted into $\int_{\Omega}\left(\Delta \omega+\lambda_{9} \omega\right) \phi_{\alpha}=0$, then passing to the limit as $s \rightarrow 0$, we have that 


$$
A_{i}\left(\sigma \int_{\Omega} \phi_{\alpha}^{2} \pm 3 \int_{\Omega} \phi_{\alpha}^{4}+\lim _{s \rightarrow 0} \frac{\mu_{i}(s)}{s^{2}} \int_{\Omega} \phi_{\alpha}^{2}\right)=0 .
$$

Similarly, testing the equation against $\psi_{\alpha}$ we get

$$
B_{i}\left(\sigma \int_{\Omega} \psi_{\alpha}^{2} \pm 3 \int_{\Omega} \phi_{\alpha}^{2} \psi_{\alpha}^{2}+\lim _{s \rightarrow 0} \frac{\mu_{i}(s)}{s^{2}} \int_{\Omega} \psi_{\alpha}^{2}\right)=0
$$

For the two above-mentioned results, making use of double integral calculation we have

$$
\int_{\Omega} \phi_{\alpha}^{2}=\frac{\pi^{2}}{2}, \int_{\Omega} \phi_{\alpha}^{4}=\frac{9}{32} \pi^{2}+\frac{3}{64} \pi^{2} \sin ^{2} 2 \alpha, \int_{\Omega} \psi_{\alpha}^{2}=\frac{\pi^{2}}{2}, \int_{\Omega} \phi_{\alpha}^{2} \psi_{\alpha}^{2}=\frac{\pi^{2}}{8}-\frac{3}{64} \pi^{2} \sin ^{2} 2 \alpha ;
$$

Now we have to distinguish between branches.

Case 1: If $\alpha=0$ or $\alpha=\frac{\pi}{2}$, it's easy to see that $\int_{\Omega} \phi_{\alpha}^{2}=\frac{\pi^{2}}{2}, \int_{\Omega} \phi_{\alpha}^{4}=\frac{9}{32} \pi^{2}, \int_{\Omega} \psi_{\alpha}^{2}=\frac{\pi^{2}}{2}, \int_{\Omega} \phi_{\alpha}^{2} \psi_{\alpha}^{2}=\frac{\pi^{2}}{8}$. For $k>0$, we have $\sigma=-\frac{9}{16}$ according to Theorem 1.From this, these equalities (3) and (4) read

$$
A_{i}\left(\lim _{s \rightarrow 0} \frac{\mu_{i}(s)}{s^{2}}+\frac{9}{8}\right)=0, B_{i}\left(\lim _{s \rightarrow 0} \frac{\mu_{i}(s)}{s^{2}}+\frac{3}{16}\right)=0
$$

For $k<0$, we have $\sigma=\frac{9}{16}$ from Theorem 1.Likewise these equalities (3) and (4) transform into

$$
A_{i}\left(\lim _{s \rightarrow 0} \frac{\mu_{i}(s)}{s^{2}}-\frac{9}{8}\right)=0, B_{i}\left(\lim _{s \rightarrow 0} \frac{\mu_{i}(s)}{s^{2}}-\frac{3}{16}\right)=0 .
$$

Since $A_{i}^{2}+B_{i}^{2}=1, A_{i}$ and $B_{i}$ are never both 0 .Thus we obtain that, say, $A_{i}=0$ or $B_{i}=0$ for both $i=1,2$;

Hence for $s$ small, the eigenvalues $\mu_{1}(s)$ and $\mu_{2}(s)$ are negative for $k>0$, also they are positive for

$k<0$.In addition, we know the first eight eigenvalues are negative from the previous discussion. To summarize, the first two branches of the bifurcation diagram, corresponding to $\alpha=0, \frac{\pi}{2}$, have Morse index ten for $k>0$, and eight for $k<0$.

Case 2: If $\alpha=\frac{\pi}{4}$ or $\alpha=\frac{3}{4} \pi$, it's easy to see $\int_{\Omega} \phi_{\alpha}^{2}=\frac{\pi^{2}}{2}, \int_{\Omega} \phi_{\alpha}^{4}=\frac{21}{64} \pi^{2}, \int_{\Omega} \psi_{\alpha}^{2}=\frac{\pi^{2}}{2}, \int_{\Omega} \phi_{\alpha}^{2} \psi_{\alpha}^{2}=\frac{5}{64} \pi^{2}$.

For $k>0$, we have $\sigma=-\frac{21}{32}$ according to Theorem 1.From this, these equalities (3) and (4) read

$$
A_{i}\left(\lim _{s \rightarrow 0} \frac{\mu_{i}(s)}{s^{2}}+\frac{21}{16}\right)=0, B_{i}\left(\lim _{s \rightarrow 0} \frac{\mu_{i}(s)}{s^{2}}-\frac{3}{16}\right)=0
$$

For $k<0$, we have $\sigma=\frac{21}{32}$ from Theorem 1.Likewise these equalities (3) and (4) transform into

$$
A_{i}\left(\lim _{s \rightarrow 0} \frac{\mu_{i}(s)}{s^{2}}-\frac{21}{16}\right)=0, B_{i}\left(\lim _{s \rightarrow 0} \frac{\mu_{i}(s)}{s^{2}}+\frac{3}{16}\right)=0 .
$$

Through a discussion similar to case 1,we learn that for $s$ small, both $k>0$ and $k<0$ there is a 
positive eigenvalue and a negative one, so the number of negative eigenvalues is 9.In other words the latter ones, corresponding to $\alpha=\frac{\pi}{4}, \frac{3}{4} \pi$ have Morse index nine, so we get the conclusion of the second half of the theorem.

\section{Conclusion}

Based on the analysis, we have that four non-degenerate bifurcation solutions from double eigenvalues are unstable. Accordingly we have a better understanding for the bifurcating problems stemming from the double eigenvalues in theory.

\section{Acknowledgements}

In this paper, the research was supported by Science and Technology Research Project of Jiangxi Provincial Department of Education (No. GJJ151250, GJJ161244), Startup Foundation for Doctors of Nanchang Normal University (No. 2015027, 2015026), Nature Science Foundation of China (No. 11661057).

\section{References}

[1] Chow SN, Hale JK. Methods of bifurcation theory [M]. New York: Springer-Verlag, 1982.

[2] Crandall MG, Rabinowitz PH. Bifurcation from simple eigenvalues [J]. Journal of Functional Analysis, 1971, 8(2): 321-340.

[3] Del Pino M, García-Melián J, Musso M. Local bifurcation from the second eigenvalue of the Laplacian in a square [J]. Proceedings of the American Mathematical Society, 2003, 131(11): 3499-3505.

[4] Shi Junping,Wang Junping.Morse indices and Exact multiplicity of solutions to Semilinear Elliptic Problems[J].Proceedings of the American Mathematical Society,1999,(127):3685-3695.

[5] Zhang Yupei,He Xibing,Local Bifurcation of Steady State Solutions for A Class of ReactionDiffusion System[J].Advances in Engineering Research,2017,(123):1673-1677. 